\title{
Lack of an effect of protease inhibitor use on glucose tolerance during pregnancy
}

\author{
Mara J. Dinsmoor and Scott T. Forrest \\ Department of Obstetrics and Gynecology, Medical College of Virginia Hospitals and Physicians of the \\ Virginia Commonwealth University Health Care System, Richmond, VA
}

\begin{abstract}
Objective: We hypothesized that HIV-positive women on protease inhibitors (PIs) would be more likely to have an elevated glucola test result than those not on PIs.

Methods: We reviewed our database of all HIV-positive pregnant women seen at our hospital. Serum glucose was measured I hour following a 50-g glucola load, at approximately 26-28 weeks of gestation. Statistical analysis was performed using Student's $t$-test, Fisher's exact test, and the Mann-Whitney rank sum test.

Results: Forty-one HIV-infected pregnant women with glucola testing were seen between January I, 1997 and March I, 2000. Fourteen patients were on Pls at the time of glucola. One patient in each group had an abnormal glucola test result (glucose $\geq 140 \mathrm{mg} / \mathrm{dl}$ ); both had normal 3-hour glucose tolerance tests. The glucola test results were similar between the PI-exposed and unexposed, with a mean difference of $5.8 \mathrm{mg} / \mathrm{dl}$ ( $95 \%$ confidence interval 9.2-20.8 mg/dl). Two neonates (both exposed to PI) had hypoglycemia (glucose $<40 \mathrm{mg} / \mathrm{dl}$ ).

Conclusions: The use of Pls does not significantly increase the risk of an elevated glucola result, nor is the mean glucola result increased in the women on Pls. The finding of hypoglycemia in neonates exposed to Pls merits further investigation.
\end{abstract}

Key words: HiV infection in Pregnancy, Gestational Diabetes, Antiretroviral Therapy, Combination Therapy, Hyperglycemia, Neonatal Hypoglycemia

\section{INTRODUCTION}

Beginning in January 1998, the Public Health Service Task Force recommended that pregnant women infected with HIV who had been receiving antiretroviral therapy prior to pregnancy should continue treatment during pregnancy ${ }^{1}$. Additionally, pregnant women infected with HIV who have had no prior antiretroviral therapy should be counseled on the use of combination therapy during pregnancy. Combination therapy, which often consists of two nucleoside analogue reverse transcriptase inhibitors and a protease inhibitor (PI), is currently the typical treatment of choice for HIV-infected adults who are not pregnant ${ }^{2,3}$. Treatment recommendations continue to evolve, however, with many experts also recommending 'protease-sparing' regimens ${ }^{2,3}$.

Although phase I trials of several PIs in pregnant HIV-infected women are ongoing, there is currently very little data regarding the safety and tolerance of PIs in pregnant women or neonates. Hyperglycemia and overt diabetes are welldocumented side-effects that have occurred with several antiretroviral medications, particularly the PIs. The mechanism of PI-induced hyperglycemia is thought to be an increase in peripheral insulin-resistance ${ }^{4,5}$. The onset of hyperglycemia

Correspondence to: Mara J. Dinsmoor, MD, Department of Obstetrics and Gynecology, Evanston Northwestern Healthcare, Northwestern University Medical School, 2650 Ridge Ave, Room 1600 WH, Evanston, IL 60201, USA.

Email: mdinsmoor@enh.org 
following PI exposure is variable with reported ranges to be from 1 month to over 1 year. Pregnancy is a state of increased insulin resistance, and glucose intolerance - or gestational diabetes complicates approximately $2 \%$ of pregnancies ${ }^{6}$. In the United States, the diagnosis of gestational diabetes is usually made by first performing a 1-hour post-glucola load as a screening test in the late second or early third trimester of pregnancy. When abnormal results are obtained for the 1-hour test, a 3-hour glucola test is performed. It was our hypothesis that the use of PIs in pregnancy may exacerbate this insulin resistance, resulting in an increased risk of hyperglycemia and gestational diabetes.

\section{SUBJECTS AND METHODS}

A prospectively maintained database was retrospectively reviewed for all HIV-infected pregnant women cared for at the Medical College of Virginia hospitals between January 1997, when PI use began, and March 2000. All patients were routinely offered antepartum, intrapartum, and neonatal zidovudine therapy based on the results of the ACTG 076 trial $^{7}$. Beginning in 1998, zidovudine monotherapy was discouraged and those women not desiring or not candidates for combination therapy with PI were offered dual therapy with zidovudine and lamivudine. Patients receiving antiretroviral therapy prior to pregnancy were counseled to continue this same therapy during pregnancy with the addition of zidovudine if they were not already taking it. Selected patients not receiving antiretroviral therapy prior to pregnancy were offered combination therapy, usually consisting of two nucleoside reverse transcriptase inhibitors and a PI. These patients generally had HIV RNA plasma viral loads greater than 5-10 000 or CD4 counts lower than 500, as per the contemporary recommendations of the US Department of Health and Human Services Panel on Clinical Practice for the Treatment of HIV Infection ${ }^{1}$. Those patients who were noncompliant with past therapy or who were non-compliant with obstetric care were generally not offered PI-containing combination therapy.

At approximately 26-28 weeks' gestation, serum glucose levels were measured 1 hour following an oral load of $50 \mathrm{~g}$ glucose in the form of glucola. If the serum glucose was $\geq 140 \mathrm{mg} / \mathrm{dl}$, the patient subsequently underwent a 3-hour glucose tolerance test. A fasting glucose level was obtained and then serum glucose levels were measured 1, 2 and 3 hours following a 100-g glucose load. Normal values were defined as $<105,190,165$, and $145 \mathrm{mg} / \mathrm{dl}$ respectively. If two or more values were abnormal, the patient was classified as a gestational diabetic ${ }^{6}$. HIV RNA plasma viral loads and CD4 counts were obtained at the first prenatal visit and at approximately 3-month intervals until delivery. In most cases, increases in the plasma viral load prompted re-evaluation and modification of the patient's antiretroviral regimen.

Neonatal glucose levels were measured only if the baby showed clinical signs of hypoglycemia, as determined by medical or nursing nursery staff. Hypoglycemia was defined by a glucose value of $<40 \mathrm{mg} / \mathrm{dl}$. Neonatal HIV infection was evaluated by performing quantitative PCR for HIV RNA on neonatal blood at birth, 2 weeks, 6 weeks and 6 months, and HIV antibody testing at 15 months of age. Neonatal HIV infection was diagnosed if any HIV RNA was detected, or if levels of anti-HIV IgG antibody remained detectable at 15 months of age.

Statistical analysis was performed using SPSS, version 10.0 (SPSS, Chicago, IL). A Fisher's exact test was performed on discrete variables, and either a Student's $t$-test (for normally distributed variables) or a Mann-Whitney rank sum test (for those not normally distributed) was performed on continuous variables. A $p$-value of $<0.05$ was considered significant.

\section{RESULTS}

At the Medical College of Virginia, we cared for 45 HIV-infected pregnant women with continuing pregnancies between January 1, 1997 and March 1, 2000. Twenty-three of the 45 patients were prescribed combination therapy including a PI prior to their delivery. Nine of these 23 patients conceived while taking combination therapy and continued therapy for the duration of the pregnancy. The median gestational age at which combination therapy was initiated in the remaining 
Table I Characteristics of HIV-infected pregnant women at the time of glucola testing, comparing those taking protease inhibitors $(\mathrm{PI})$ and those who were not $(\mathrm{No} \mathrm{PI})$

\begin{tabular}{|c|c|c|c|}
\hline & $P I(n=14)$ & No $P I(n=27)$ & p-value \\
\hline Mean age, years $\pm S D$ & $30.1 \pm 6.4$ & $26.3 \pm 4.7$ & 0.04 \\
\hline \multicolumn{4}{|l|}{ Ethnic origin, $n(\%)$} \\
\hline African-American & $12(86)$ & $21(78)$ & NS \\
\hline Caucasian & $2(14)$ & $6(22)$ & NS \\
\hline AIDS, number affected (\%) & $4(29)$ & $5(19)$ & NS \\
\hline Mean CD4 count, cells $/ \mathrm{mm}^{3} \pm \mathrm{SD}$ & $401 \pm 170$ & $465 \pm 293$ & NS \\
\hline Median viral load, copies/ml (range) & $1022(0-70300)$ & $2790(0-88000)$ & NS \\
\hline Mean gesatational age, weeks $\pm S D$ & $27.2 \pm 1.4$ & $27.7 \pm 3$ & NS \\
\hline Mean glucola result, $\mathrm{mg} / \mathrm{dl} \pm \mathrm{SD}$ & $108 \pm 25$ & $102 \pm 21$ & NS \\
\hline Abnormal glucola ( $\geq 140 \mathrm{mg} / \mathrm{dl}), n(\%)$ & I (7) & I (4) & NS \\
\hline
\end{tabular}

SD, standard deviation; NS, not significant

14 patients was 27 weeks (range 15-35 weeks.) Eight patients on combination therapy started in the third trimester after glucola screening had been completed. Twenty-two of the 45 patients were not prescribed combination therapy (five declined, ten were known to be non-compliant with previously prescribed medications, four had low viral load counts, two had minimal prenatal care, and one had failed PI therapy in the past). Generally, these patients were prescribed zidovudine with lamivudine added in some cases, as described earlier. One patient declined all antiretroviral therapy during her pregnancy, but accepted intrapartum zidovudine and nevirapine.

Table 1 describes the demographic characteristics of both groups. There were no significant differences in race or gestational age between the two groups at the time of glucola screening. Those patients who were prescribed PI-containing combination therapy tended to have lower CD 4 counts and lower viral loads, although these trends were not statistically significant. Four patients (one on combination therapy) did not have glucola screening performed prior to delivery due to scant prenatal care. In the remaining 41 patients, there were no significant differences in the mean glucola result or in the incidence of abnormal glucola results between women taking a PI and those who were not. The mean difference in glucola test results was $5.8 \mathrm{mg} / \mathrm{dl}$ (95\% confidence interval (CI) $9.2-20.8 \mathrm{mg} / \mathrm{dl})$. No patient in either group was diagnosed with gestational diabetes. The median duration of PI use prior to glucola screening was
27 weeks (range 2-31 weeks.) Twelve patients (86\%) had been on PI for at least 2 months, and eight $(57 \%)$ had been on PI for at least 6 months. There were no significant differences in glucola results when the analysis was confined to those patients with PI exposures of 2 or 6 months prior to testing.

Of the 14 patients on PI-containing regimens, the majority (nine) were taking nelfinivir plus lamivudine and zidovudine. Other combinations included indinivir plus lamivudine and zidovudine (two patients), nelfinivir plus zidovudine and nevirapine (one), nelfinivir plus lamivudine and stavudine (one), and saquinivir plus lamivudine and stavudine (one patient).

As shown in Table 2, there were no significant differences between the PI-exposed and unexposed patients in gestational age or birth weight at the time of delivery, and only one infant exposed to PI was born prematurely, at 36 weeks. Two infants exposed to Nelfinivir were diagnosed with symptomatic hypoglycemia (serum glucose levels of 26 and $35 \mathrm{mg} / \mathrm{dl}$, respectively) in the nursery. Only two unexposed infants exhibited symptoms of hypoglycemia and were tested; neither was found to be hypoglycemic. No other metabolic abnormalities were seen in the PIexposed neonates nor were any congenital anomalies or dysmorphology noted. The two babies with hypoglycemia were treated symptomatically and did well. These differences remained non-significant when patients exposed to PI only in the third trimester (seven patients) were 
Table 2 Neonatal outcomes, comparing those mothers taking a protease-inhibitor-containing regimen (PI) at the time of glucola testing to those who were not (No PI)

\begin{tabular}{lcc}
\hline & $P I(n=14)$ & No PI $(n=27)$ \\
\hline Mean gestational age, weeks \pm SD & $38.4 \pm 1.6$ & $38.3 \pm 1 . I$ \\
Mean birth weight, g \pm SD & $3065 \pm 553$ & $3159 \pm 43$ I \\
Mode of delivery, $n$ (\%) & & \\
$\quad$ Vaginal & $2(14)$ & $9(33)$ \\
Cesarean section (indicated) & $4(29)$ & $7(26)$ \\
Cesarean section (scheduled) & $8(57)$ & $1 \mathrm{I}(4 \mathrm{I})$ \\
Neonatal hypoglycemia, $n(\%)$ & $2(14)$ & 0 \\
Neonatal HIV infection, $n(\%)$ & 0 & $3(1 \mathrm{I})$ \\
\hline
\end{tabular}

p-values were not significant for all camparisons

included in the PI-exposed group (data not shown).

\section{DISCUSSION}

In our patient population, the use of PIs in pregnancy does not significantly increase the risk of having an elevated glucola value nor does it increase the risk of gestational diabetes. The small sample size and relatively short duration of PI exposure are limitations that may increase the chance of a beta error. Given that the 95\% CI for the difference between the two groups is $-9.2-20.8$, our results show that if there is an increase in glucose intolerance, the magnitude of the effect is likely not clinically significant. In addition, hyperglycemia and dyslipidemias have been reported following only 1 month of PI exposure. It has been postulated that the peripheral insulin resistance seen in these patients may be a result of PI binding to cytoplasmic retinoicacid-binding protein type 1 and low-density lipoprotein-receptor-related protein, and the resultant hyperlipidemia ${ }^{8}$. Hadigan and colleagues reported that HIV-infected women, even in the absence of antiretroviral therapy, had hyperinsulinemia and hypertriglyceridemia compared to HIV-negative women?. Although we did not detect an increased incidence of glucose intolerance in our HIV-infected pregnant population, baseline hyperinsulinemia and insulin resistance as a result of the pregnancy could potentially mask any further increases in insulin resistance due to PI use. A potential confounder is the fact that many of the patients who were prescribed PIs had more advanced HIV disease than the women in the comparison group.

We did not measure lipid levels or insulin resistance directly as has been done in other studies, but searched for sequelae of increased insulin resistance, namely hyperglycemia and glucose intolerance. Perhaps a better indicator of maternal insulin resistance and possibly subtle hyperglycemia is the occurrence of neonatal hypoglycemia in two of the PI-exposed neonates. Fetal beta-cell hyperplasia following exposure to maternal hyperglycemia, leading to an exaggerated insulin release and resulting in postnatal hypoglycemia, as seen following delivery in neonates of diabetic mothers, is a possible explanation. However, a direct effect of placentally transferred PI cannot be excluded. As practitioners gain experience and increasingly prescribe the use of novel antiretroviral therapies during pregnancy, surveillance for subtle and delayed effects, such as neonatal hypoglycemia, should be continued.

\section{REFERENCES}

1. Public Health Service Task Force recommendations for the use of antiretroviral drugs in pregnant women infected with HIV-1 for maternal health and for reducing perinatal HIV-1 transmission in the United States. Centers for Disease Control and Prevention. Morb Mortal Wkly Rep 1998;47(RR-2):1-30 
2. Department of Health and Human Services and the Henry J. Kaiser Family Foundation, Panel on Clinical Practices for Treatment of HIV Infection. Guidelines for the use of antiretroviral agents in HIV-infected adults and adolescents. HIV Clin Trials 2000;1:60-110

3. Carpenter CCJ, Cooper DA, Fischl MA, et al. Antiretroviral therapy in adults: updated recommendations of the International AIDS Society-USA Panel. J Am Med Assoc 2000;283:381-90

4. Carr A, Samaras K, Thorisdottir A, et al. Diagnosis, prediction, and natural course of HIV-1 proteaseinhibitor-associated lipodystrophy, hyerlipidaemia, and diabetes mellitus: a cohort study. Lancet 1999; 353:2093-9.

5. Walli R, Herfort O, Michl GM, et al. Treatment with protease inhibitors associated with peripheral insulin resistance and impaired oral glucose tolerance in HIV-1-infected patients. AIDS 1998;12:F167-73

ReCEIVED 11/20/02; ACCEPTED 06/20/02
6. Landon MB. Diabetes mellitus and other endocrine diseases. In Gabbe SG, Niebyl JR, Simpson JL, eds. Obstetrics: Normal and Problem Pregnancies, 3rd edn. New York: Churchill-Livingstone, 1996;1037-81

7. Connor EM, Sperling RS, Gelber R, et al. Reduction of maternal-infant transmission of human immunodeficiency virus type I with zidovudine treatment. N Engl J Med 1994;331:1173-80

8. Carr A, Samaras K, Chisholm DJ, Cooper DA. Pathogenesis of HIV-1-protease inhibitor-associated peripheral lipodystrophy, hyerlipidaemia, and insulin resistance. Lancet 1998;351:1881-3

9. Hadigan C, Miller K, Corcoran C, et al. Fasting hyperinsulinemia and changes in regional body composition in human immunodeficiency virusinfected women. J Clin Endocrinol Metab 1999;84: $1932-7$ 


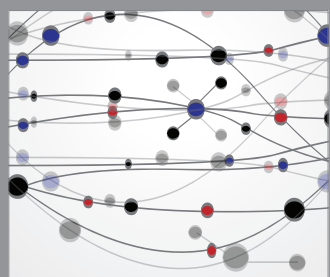

The Scientific World Journal
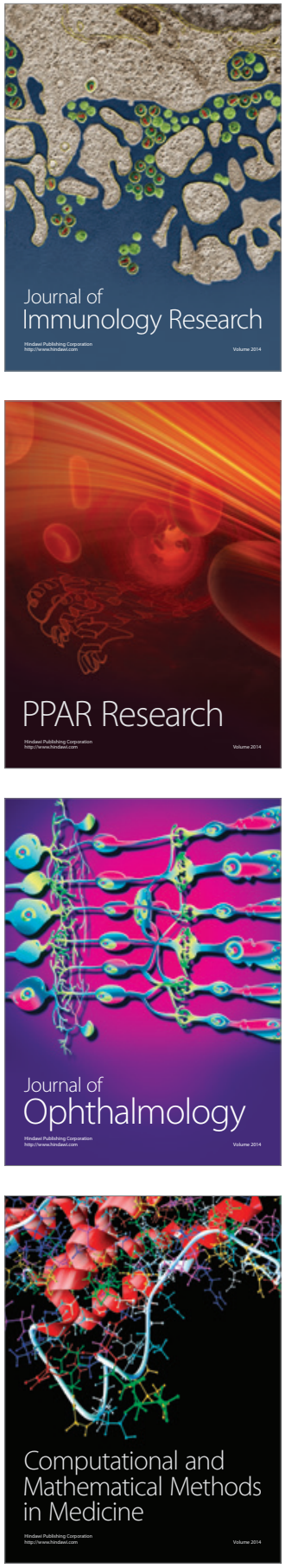

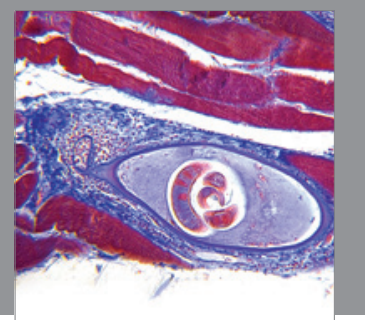

Gastroenterology

Research and Practice
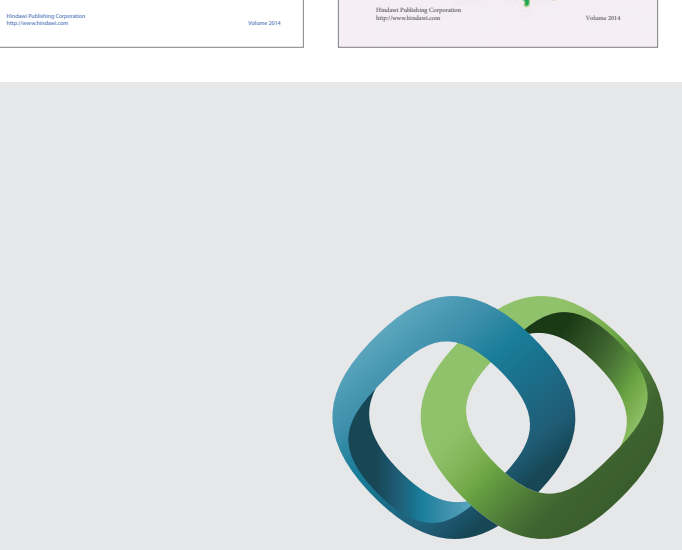

\section{Hindawi}

Submit your manuscripts at

http://www.hindawi.com
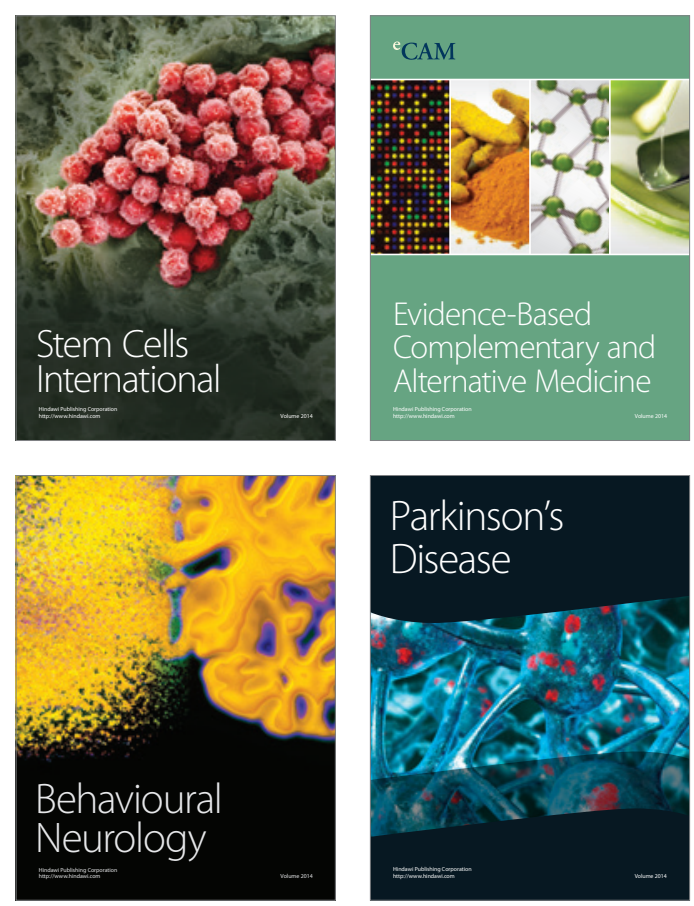

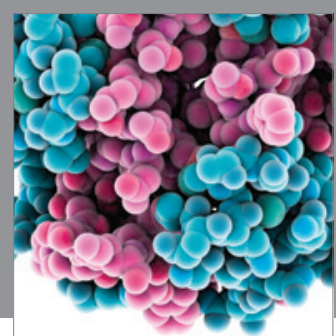

Journal of
Diabetes Research

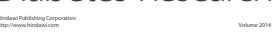

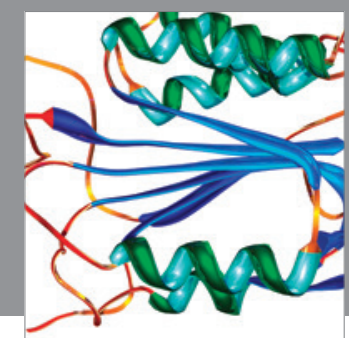

Disease Markers
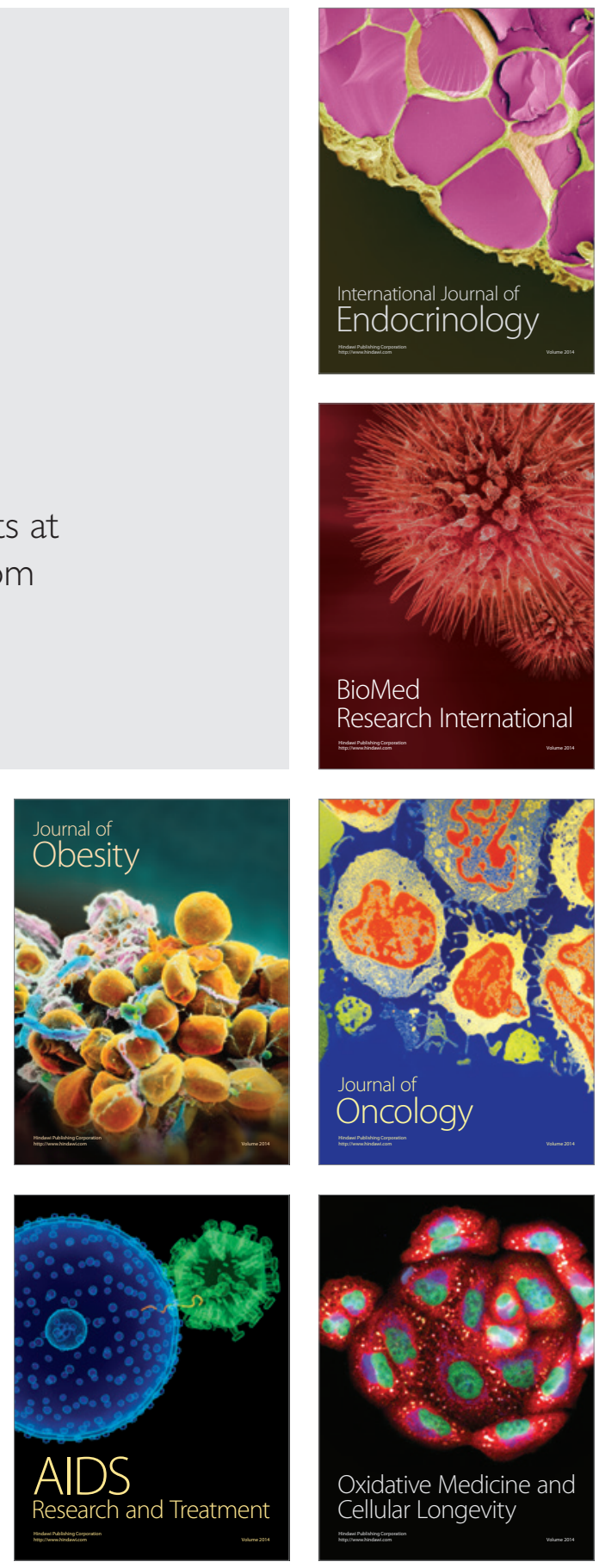